\title{
Improving the cytoplasmic maturation of bovine oocytes matured in vitro with intracellular and/or extracellular antioxidants is not associated with increased rates of embryo development
}

\author{
Nathália A.S. Rocha-Frigoni, Beatriz C.S. Leão, Priscila Chediek Dall'Acqua, \\ Gisele Z. Mingoti*
}

Laboratory of Reproductive Physiology, Department of Animal Health, School of Veterinary Medicine, UNESP-Universidade Estadual Paulista, Araçatuba, São Paulo, Brazil

Post-Graduation Program in Veterinary Medicine, Department of Animal Reproduction, School of Agrarian and Veterinarian Sciences, UNESP-Universidade Estadual Paulista, Jaboticabal, São Paulo, Brazil

\section{A R T I C L E I N F O}

\section{Article history:}

Received 5 October 2015

Received in revised form 2 June 2016

Accepted 5 June 2016

\section{Keywords:}

Oxidative status

Oocyte competence

Intracellular antioxidants

Extracellular antioxidants

Mitochondrial potential

\begin{abstract}
A B S T R A C T
The production of reactive oxygen species (ROS) is a normal process that occurs in the cellular mitochondrial respiratory chain. However, an increase in ROS levels during in vitro production of bovine embryos induces oxidative stress, leading to failed embryonic development. Therefore, we investigated whether supplementation of IVM medium with intracellular (cysteine and cysteamine; $C+C$ ) and/or extracellular (catalase; CAT) antioxidants improves the culture system, affects the mitochondrial membrane potential, affects the intracellular levels of ROS and glutathione (GSH) in the bovine oocytes at the end of maturation, and thereby affects the subsequent embryonic development. At the end of IVM, the metaphase II rates were unaffected by the treatments $(76.7 \pm 1.7 \%$ to $80.6 \pm 5.2 \%$; $>0.05)$. The intracellular ROS levels, expressed in arbitrary fluorescence units, found in the oocytes treated with intracellular antioxidants $(C+C$ and $C+C+C A T$ groups; 1.06 , averaged $)$ were as low as those observed in immature oocytes ( 0 hour: $1.00 \pm 0.12)$. Among mature oocytes, higher $(\mathrm{P}<0.05)$ ROS levels were found in the control group $(1.91 \pm 0.10)$ when compared to the ROS levels found in oocytes treated with antioxidants. Intracellular GSH levels in all groups were lower $(0.17 \pm 0.09$ to $0.51 \pm 0.05 ; \mathrm{P}<0.05)$ than those in immature oocytes $(1.00 \pm 0.08)$, although GSH levels in the $C+C$ group $(0.51 \pm 0.05)$ were greater $(P<0.05)$ than in the control, CAT, and C + C + CAT groups (0.23; averaged). The mitochondrial membrane potential in all groups was improved (1.6; averaged; $\mathrm{P}<0.05$ ) compared to the membrane potential observed in the immature oocytes $(1.00 \pm 0.05)$, with the exception of the $C+C$ group $(0.94 \pm 0.03)$. There was no effect $(P>0.05)$ of antioxidant supplementation on embryonic development to the blastocyst stage (36.1\%; averaged); however, there was an increased tendency $(\mathrm{P}=0.0689)$ to obtain a higher blastocyst rate for the $\mathrm{C}+\mathrm{C}+$ CAT group $(47.5 \pm 5.6 \%)$ compared to the control group $(29.9 \pm 4.8 \%)$. In conclusion, despite improvements in specific parameters of cytoplasmic maturation, the addition of intracellular and/or extracellular antioxidants during IVM did not affect embryo development.
\end{abstract}

(c) 2016 Elsevier Inc. All rights reserved.

\footnotetext{
* Corresponding author. Tel.: +55 183636 1375; fax: +55 1836361352 .

E-mail address: gmingoti@fmva.unesp.br (G.Z. Mingoti).
} 


\section{Introduction}

Many attempts have been made to increase the in vitro production (IVP) of cattle embryos by improving the conditions of the culturing systems, especially during IVM. IVM constitutes the in vitro advancement of an oocyte from the diplotene stage of prophase I (germinal vesicle [GV]) to metaphase II (MII) and cytoplasmic maturation [1]. This is a crucial step because in vitro-matured oocytes exhibit inferior acquisition of developmental competence compared to those matured in vivo. Therefore, during maturation, the oocytes acquire the developmental capacity, i.e., their intrinsic ability to support the subsequent stages of fertilization and early embryo development [2]. Many studies have reported that the culture environment to which oocytes and embryos are exposed can affect their quality [3-6]. In addition, among the various factors that can influence the culture environment, the oxidative stress that is induced by the greater oxygen tension has received special attention in the last decade [7]. The oxygen tension routinely used in IVM culture systems is often higher than that found in the microenvironment of the female reproductive tract ( $\sim 20 \%$ and $3 \%-9 \% \mathrm{O}_{2}$, respectively), and such high oxygen tension is believed to induce the excessive generation of reactive oxygen species (ROS), namely, hydrogen peroxide $\left(\mathrm{H}_{2} \mathrm{O}_{2}\right)$, hydroxyl radical, and peroxyl radical. In addition to increased oxygen tension, other exogenous factors capable of inducing ROS generation can be found in the culture medium itself, including traces of metallic cations (such as $\mathrm{Fe}$ and $\mathrm{Cu}$ ) that are often present in the water and/or chemical products used to prepare the culture medium, and amine oxidase, which is found in serum $[8,9]$.

Reactive oxygen species production is a normal process that occurs within cells, including embryos, cumulus cells, and oocytes [8]. Under physiological conditions, the main source of ROS is oxidative phosphorylation in the mitochondria; ROS are produced through the electron transfer reaction to oxygen [10]. Although ROS are involved in many physiological processes related to ovarian activity and gametogenesis [11] as well as in the fertilization capacity of bovine spermatozoa and in the interaction between spermatozoa and oocytes [12], oxidative stress occurs when ROS production exceeds the cellular antioxidant defense mechanisms, resulting in disturbances in reduction/oxidation (redox)-regulated processes. These radicals are extremely reactive and unstable and therefore may interact with several molecules to acquire electrons in an attempt to become stable [13]. These interactions may, in turn, induce a cascade of chain reactions that can eventually lead to cellular damage, including lipid peroxidation (mainly membrane phospholipids) and the oxidation of amino acids and nucleic acids $[8,14,15]$. In addition, high levels of ROS cause DNA damage because of the disruption of the mitochondrial membranes and lead to the consequent release of cytochrome $C$ and activation of caspase cascades. These events culminate in apoptosis and failed embryonic development [16]. Thus, oxidative stress may decrease the viability of in vitro-produced embryos [17].

To circumvent these harmful effects, biological systems have developed mechanisms to control ROS levels, including enzymatic (superoxide dismutase, glutathione [GSH] peroxidase, and catalase) and nonenzymatic antioxidant agents ( $\alpha$-tocopherol, ascorbic acid, $\beta$-carotene, and GSH, among others) [18]. Thus, given the constant threat to cell viability imposed by the action of ROS, these antioxidants have been applied to the IVP culture systems used to produce bovine embryos, with the goal of improving the production and quality of oocytes and embryos. Reports in the literature regarding the effects of supplementing media with extracellular enzymatic antioxidants, such as catalase, are very controversial $[19,20]$. However, a recent report demonstrated that the addition of catalase during IVP reduced intracellular ROS levels and the rate of apoptosis in bovine embryos [5]. This finding inspired further studies focused on the modulation of extracellular ROS production to improve IVP systems.

More attention has been given to the supplementation of IVM medium with thiol compounds such as cysteine or cystine [21] and cysteamine [13], which increase intracellular GSH levels in bovine oocytes. Alterations in GSH concentration and redox status have been associated with the oxidative stress induced in the mitochondria by peroxides and other oxidants, as reviewed by Lash [22]. Thus, GSH acts as the main nonenzymatic defense system in both oocytes and embryos and promotes both the detoxification of lipid peroxides and thiol proteins and the removal of $\mathrm{H}_{2} \mathrm{O}_{2}$ [18]. The biosynthesis of GSH is limited by the availability of cysteine in the medium; however, this substance is unstable outside the cell. Cysteine is likely absent from the culture medium because of its auto-oxidation to cystine [23]. Low-molecular-weight thiol compounds, such as cysteamine and $\beta$-mercaptoethanol, promote the reduction of cystine to cysteine, which enhances cysteine uptake and increases GSH synthesis [13].

In the present study, we hypothesized that the improved quality of oocytes at the end of maturation, e.g., by increasing GSH levels and mitochondrial function, could increase oocyte acquisition of development potential. Therefore, we evaluated the effects of intracellular (cysteine and cysteamine) and/or extracellular (catalase) antioxidants during IVM on the oocyte quality and its acquisition of developmental capacity. Specifically, we assessed the following parameters: the meiosis progression to MII stage, the mitochondrial membrane potential and the intracellular levels of ROS and GSH in bovine oocytes at the end of maturation, and the embryonic development to the blastocyst stage.

\section{Materials and methods}

\subsection{Chemicals, reagents, and media}

Chemicals were purchased from Sigma Chemical Co. (St Louis, MO, USA) unless otherwise stated. The medium for IVM consisted of TCM199 (Gibco; Invitrogen Co., Grand Island, NY, USA) supplemented with 10\% (v:v) fetal calf serum (Gibco, Invitrogen Co.), $0.2 \mathrm{mM}$ sodium pyruvate, $25 \mathrm{mM}$ sodium bicarbonate, $50 \mu \mathrm{g} / \mathrm{mL}$ amikacin, $0.5 \mu \mathrm{g} / \mathrm{mL}$ FSH (Folltropin-V; Bioniche Animal Health, Ontario, Canada), and $100 \mathrm{IU} / \mathrm{mL}$ hCG (Vetecor; Hertape Calier, Juatuba, MG, Brazil). The medium for IVF consisted of 
Tyrode's albumin lactate pyruvate (TALP) containing $0.2 \mathrm{mM}$ Na-pyruvate, $6 \mathrm{mg} / \mathrm{mL}$ fraction $\mathrm{V}$ fatty acid-free BSA, $25 \mathrm{mM}$ sodium bicarbonate, $13 \mathrm{mM}$ Na-lactate, $50 \mu \mathrm{g} / \mathrm{mL}$ amikacin, $40 \mu \mathrm{L} / \mathrm{mL}$ PHE solution (1 mM hypotaurine, $2 \mathrm{mM}$ penicillamine, and $250 \mu \mathrm{M}$ epinephrine), and $10 \mu \mathrm{g} / \mathrm{mL}$ heparin, as previously described [24]. The medium for the IVC of embryos consisted of modified synthetic oviductal fluid (SOF), as previously described [25], supplemented with $50 \mu \mathrm{g} / \mathrm{mL}$ amikacin, $5 \mathrm{mg} / \mathrm{mL}$ BSA, and $2.5 \%(\mathrm{v}: \mathrm{v})$ fetal calf serum.

Antioxidants were diluted to the appropriate concentration according to the experiment in TCM199 medium: cysteine (C7352) was freshly diluted each day of the experiment to obtain a final concentration of $0.6 \mathrm{mM}$; millimolar stock concentrations (50 mM) of cysteamine (M9768) were stored at $-20^{\circ} \mathrm{C}$ dissolved in TCM199 up to 1 week and were freshly diluted for each experiment to obtain a final concentration of $100 \mu \mathrm{M}$; a stock solution (2000 IU/mL) of catalase (C1345) was stored at $4{ }^{\circ} \mathrm{C}$ dissolved in TCM199 up to 1 week and was freshly diluted for each experiment to obtain a final concentration of $100 \mathrm{IU} / \mathrm{mL}$.

\subsection{Oocyte collection and maturation}

Ovaries from slaughtered cows were obtained in a local abattoir and transported to the laboratory. Intact cumulusoocyte complexes (COCs) were aspirated from antral follicles (3-8 $\mathrm{mm}$ in diameter), and oocytes with at least four layers of cumulus cells with homogenous cytoplasm were selected for the experiments. Selected COCs were washed and cultured in IVM medium with or without antioxidant supplementation as follows: (1) $0.6 \mathrm{mM}$ cysteine combined with $100 \mu \mathrm{M}$ cysteamine ( $\mathrm{C}+\mathrm{C}$ group); (2) 100 IU catalase (CAT group); (3) $0.6 \mathrm{mM}$ cysteine combined with $100 \mu \mathrm{M}$ cysteamine and 100 IU catalase (C + C + CAT group); or (4) without supplementation (control). The IVM culturing was performed in $500 \mu \mathrm{L}$ of medium without mineral oil (25 oocytes per well) in a four-well cell culture cluster (NUNC, Nunclon Delta Treated 4-Well IVF Dish, Denmark) for 22 hours at $38.5{ }^{\circ} \mathrm{C}$ in an atmosphere of $5 \% \mathrm{CO}_{2}$ in air with maximum humidity.

\subsection{Assessment of nuclear maturation}

Oocytes that were harvested immediately after their removal from the follicle ( 0 hour) and after 22 hours of IVM were stripped from their cumulus cells by vortexing in PBS with $0.1 \%$ hyaluronidase for 3 minutes. Denuded oocytes were then stained with Hoechst $33342(1 \mu \mathrm{g} / \mathrm{mL})$ for 30 minutes at room temperature to determine their maturation status. The oocytes were washed, transferred to glass slides with mounting glycerol, and observed under an inverted microscope (Olympus, IX51) at an excitation wavelength of $510 \mathrm{~nm}$ and an emission wavelength of $590 \mathrm{~nm}$. Oocytes classified as GV stage were considered immature; those with a metaphase plate and first polar body were classified as MII and were considered mature. Oocytes in stages of maturation between GV and MII (metaphase I, anaphase I, and telophase I) were considered to be in intermediate (INT) stages of meiosis.

\subsection{Quantification of intracellular ROS levels by dichlorofluorescein assay}

Intracellular ROS levels in oocytes were quantified immediately after their removal from the follicle ( 0 hour) and after 22 hours of IVM using the fluorescent probe 6-carboxi-2',7'-dichlorodihydrofluoresceine diacetate $\left(\mathrm{H}_{2} \mathrm{DCFDA}\right.$; Molecular Probes, Invitrogen, Oregon, USA). A stock solution of $\mathrm{H}_{2}$ DCFDA dissolved in DMSO was diluted in PBS to a working concentration of $5 \mu \mathrm{M}$ [14]. Oocytes were washed twice in PBS and placed into fourwell cell culture plates containing $500 \mu \mathrm{L}$ of $5 \mu \mathrm{M}$ $\mathrm{H}_{2}$ DCFDA. The four-well plates were incubated at $38.5^{\circ} \mathrm{C}$ in a dark, humidified, $5 \% \mathrm{CO}_{2}$ atmosphere for 30 minutes. Stained oocytes were washed twice with fresh PBS and imaged immediately using an Olympus IX51 inverted microscope running Q-Capture Pro Image software (Media Cybernetics, Inc., Version 5.0.1.26) with an excitation wavelength of $495 \mathrm{~nm}$ and an emission wavelength of $520 \mathrm{~nm}$ to quantify the fluorescence signal intensities (pixels). The background signal intensity was subtracted from the measured values of the treatment micrographs. To evaluate the improvement in cytoplasmic maturation during IVM culture, immature oocytes ( 0 hour) were chosen as the calibrator, and the measured value of each treatment micrograph was divided by the mean of the calibrator to generate relative expression levels (arbitrary fluorescence units). The means of the relative expression values were plotted graphically with error bars representing the standard error of the mean (SEM).

\subsection{Quantification of intracellular levels of reduced GSH}

Intracellular GSH levels were quantified using the fluorescent probe ThiolTracker Violet (Glutathione Detection Reagent; Molecular probes, Invitrogen, Oregon, USA) according to the manufacturer's instructions. Oocytes that were harvested immediately after their removal from the follicle ( 0 hour) and after 22 hours of IVM were washed twice in PBS and placed into four-well cell culture plates containing $500 \mu \mathrm{L}$ of $20 \mu \mathrm{M}$ ThiolTracker. The four-well plates were incubated at $38.5{ }^{\circ} \mathrm{C}$ in a dark, humidified, $5 \%$ $\mathrm{CO}_{2}$ atmosphere for 30 minutes. Stained oocytes were washed twice with fresh PBS and imaged immediately using an Olympus IX51 inverted microscope running QCapture Pro Image software with an excitation wavelength of $404 \mathrm{~nm}$ and an emission wavelength of $526 \mathrm{~nm}$ to quantify the fluorescent signal intensities (pixels). The background signal intensity was subtracted from the measured values of the treatment micrographs. Immature oocytes ( 0 hour) were chosen as the calibrator, and the measured value of each treatment micrograph was divided by the mean of the calibrator to generate relative expression levels (arbitrary fluorescence units). The means of the relative expression values were plotted graphically with error bars representing the SEM.

\subsection{Assessment of mitochondrial membrane potential ( $\Delta \Psi \mathrm{m})$}

Mitochondrial membrane potential was assessed using the fluorescent probe MitoTracker Red (CMXRos; 
Molecular Probes, Invitrogen, Oregon, USA) according to the manufacturer's instructions. Oocytes that were harvested immediately after their removal from the follicle ( 0 hour) and after 22 hours of IVM were washed twice in PBS and placed into four-well cell culture plates containing $500 \mu \mathrm{L}$ of $500-\mathrm{nM}$ MitoTracker. The four-well plates were incubated at $38.5{ }^{\circ} \mathrm{C}$ in a dark, humidified, $5 \% \mathrm{CO}_{2}$ atmosphere for 30 minutes. Stained oocytes were washed twice with fresh PBS and imaged immediately using an Olympus IX51 inverted microscope running QCapture Pro Image software with an excitation wavelength of $579 \mathrm{~nm}$ and an emission wavelength of $599 \mathrm{~nm}$ to quantify the fluorescence signal intensities (pixels). The background signal intensity was subtracted from the measured values of the treatment micrographs. Immature oocytes ( 0 hour) were chosen as the calibrator, and the measured value of each treatment micrograph was divided by the mean of the calibrator to generate relative expression levels (arbitrary fluorescence units). The means of the relative expression values were plotted graphically with error bars representing the SEM.

\subsection{In vitro fertilization and culture}

After IVM, COCs were washed three times in the control IVM medium (without antioxidant supplementation) and twice in TALP medium to completely remove any residual concentrations of antioxidants before being transferred to the fertilization droplet. Oocytes were subjected to fertilization with Nellore bull (Bos taurus indicus) frozen semen from one animal and the same cryopreservation batch. Frozen semen straws were obtained from a commercial company (Alta Genetics Brazil Ltda, Uberaba, MG, Brazil) and stored in liquid nitrogen and were thawed for each experiment according to the vendor instructions $\left(36^{\circ} \mathrm{C}\right.$ for 40 seconds). Motile spermatozoa were obtained by centrifugation of the frozen-thawed semen on a Percoll (GE Healthcare, Uppsala, Sweden) discontinuous density gradient ( $250 \mu \mathrm{L}$ of $45 \%$ Percoll over $250 \mu \mathrm{L}$ of $90 \%$ Percoll in a $1.5-\mathrm{mL}$ microtube) for 5 minutes at $\times 2500 \mathrm{~g}$ at room temperature. Sperm cells were added to the fertilization droplet at $2 \times 10^{6}$ cells $/ \mathrm{mL}$. The COCs $(25$ per $90-\mu \mathrm{L}$ droplet overlaid with mineral oil) and spermatozoa were coincubated for 18 hours at $38.5{ }^{\circ} \mathrm{C}$ in an atmosphere of $5 \% \mathrm{CO}_{2}$ in air, with maximum humidity. The day of fertilization was defined as Day 0.

After fertilization, the presumptive zygotes were partially stripped from the cumulus cells by gentle pipetting and subsequently washed three times in TALP medium and twice in IVC medium. Zygotes were transferred to $500 \mu \mathrm{L}$ of IVC medium without mineral oil (50 oocytes per well) on a monolayer of cumulus cells in a four-well cell culture plate. The culture was carried out at $38.5^{\circ} \mathrm{C}$ in an atmosphere of $5 \% \mathrm{CO}_{2}$ in air, with maximum humidity. Cleavage rates were assessed under stereoscopic microscopy at $\times 40$ magnification at 72 hours postinsemination, and blastocyst development rates were recorded at 168 hours postinsemination (Day 7).

\subsection{Experimental design}

\subsubsection{Experiment I: effects of antioxidants during IVM on} meiosis progression

Oocytes were matured for 22 hours in vitro in medium supplemented with $\mathrm{C}+\mathrm{C}(\mathrm{n}=110)$, CAT $(\mathrm{n}=104), \mathrm{C}+\mathrm{C}$ + CAT $(\mathrm{n}=102)$, or without supplementation (control; $\mathrm{n}=120$ ). Oocytes that were immediately harvested after their removal from the follicle $(0$ hour $=$ immature oocytes; $\mathrm{n}=129$ ) and mature oocytes were stained for assessment of meiosis progression to MII. Treatments were repeated in five replicates. A 1-cell culture well containing 25 oocytes was considered the experimental unit for each replicate of each group. The percentage of oocytes at specific stages of meiosis was estimated by considering the total number of oocytes in each maturation well.

\subsubsection{Experiment II: effects of antioxidants during IVM on intracellular ROS and GSH levels in bovine oocytes}

Oocytes were matured for 22 hours in vitro in medium supplemented with $\mathrm{C}+\mathrm{C}(\mathrm{n}=111)$, CAT $(\mathrm{n}=117), \mathrm{C}+\mathrm{C}+$ CAT $(n=130)$, or without supplementation (control; $\mathrm{n}=129$ ). Oocytes that were immediately harvested after their removal from the follicle $(0$ hour $=$ immature oocytes; $\mathrm{n}=132$ ) and mature oocytes were simultaneously stained with the fluorescent probes $\mathrm{H}_{2}$ DCFDA and ThiolTracker Violet for assessment of ROS and GSH levels, respectively. Treatments were repeated in five replicates. Within each replicate, the experimental unit for each group consisted of each individual stained oocyte.

\subsubsection{Experiment III: effects of antioxidants during IVM on $\triangle \Psi \mathrm{m}$ in bovine oocytes}

Oocytes were matured for 22 hours in vitro in medium supplemented with $C+C(n=130)$, CAT $(n=130), C+C+$ CAT $(\mathrm{n}=130)$, or without supplementation (control; $\mathrm{n}=130$ ). Oocytes that were immediately harvested after their removal from the follicle $(0$ hour $=$ immature oocytes; $\mathrm{n}=107)$ and mature oocytes were stained with the fluorescent probe MitoTracker Red for assessment of $\Delta \Psi \mathrm{m}$. Treatments were repeated in five replicates. Within each replicate, the experimental unit for each group consisted of each individual stained oocyte.

\subsubsection{Experiment IV: effects of antioxidants during IVM on embryo development in vitro}

Oocytes were matured for 22 hours in vitro in medium supplemented with $\mathrm{C}+\mathrm{C}(\mathrm{n}=197), \mathrm{CAT}(\mathrm{n}=183), \mathrm{C}+\mathrm{C}+$ CAT $(\mathrm{n}=195)$, or without supplementation (control; $\mathrm{n}=191$ ). Mature oocytes were then subjected to IVF, and the presumptive zygotes were cultured in SOF medium without antioxidant supplementation for up to 7 days when their development to the blastocyst stage was evaluated. Treatments were repeated in five replicates. A 1-cell culture well containing 50 embryos was considered the experimental unit for each replicate of each group. The percentage of blastocysts recovered on Day 7 after insemination was based on the number of developed blastocysts per oocyte inseminated. 


\subsection{Statistical analysis}

Data were analyzed by ANOVA using JMP statistical software version 5.0.1a (SAS Inst. Inc., Cary, NC, USA). Data in percentages were arcsine transformed before ANOVA. When a statistically significant effect was found, Tukey's test was applied for multiple comparisons of means. The level of statistical significance was set at $\mathrm{P}<0.05$, and $\mathrm{P}$ values between 0.051 and 0.1 were considered as tendency. All values are presented as means with their corresponding SEM.

\section{Results}

Data for the stages of nuclear maturation in oocytes are summarized in Table 1 . There were no significant differences $(P>0.05)$ between groups with respect to the percentages of oocytes remaining at the stages of GV $(9.4 \pm 5.6 \%$ to $14.15 \pm 5.6 \%)$ and INT $(5.5 \pm 1.8 \%$ to $12.3 \pm 3.3 \%)$ or reaching the stage of MII $(76.7 \pm 1.7 \%$ to $80.6 \pm 5.2 \%$ ) after 22 hours of IVM. A sample of oocytes were evaluated immediately after follicular puncture (0 hour, immature oocytes), and it was found that $98.4 \%$ were at the GV stage, $1.6 \%$ were at the INT stages, and $0 \%$ were at the MII (data not presented in the table).

Representative photomicrographs of stained bovine oocytes for evaluation of ROS, GSH, and $\Delta \Psi \mathrm{m}$ are shown in Figure 1.

Intracellular ROS levels (expressed as arbitrary fluorescence units; Fig. 2) for control oocytes (1.91 \pm 0.10$)$ were significantly greater $(\mathrm{P}<0.05)$ than for oocytes treated with the antioxidants $C+C(1.11 \pm 0.04)$, CAT $(1.45 \pm 0.08)$, and $\mathrm{C}+\mathrm{C}+\mathrm{CAT}(1.07 \pm 0.04)$. Among the treated oocytes, those from CAT group exhibited significantly greater $(\mathrm{P}<0.05)$ ROS levels than those from $\mathrm{C}+\mathrm{C}$ and $\mathrm{C}+\mathrm{C}+\mathrm{CAT}$ groups. There were no significant differences $(P>0.05)$ between the immature oocytes ( 0 hour; $1.00 \pm 0.12$ ) and the mature oocytes from the $\mathrm{C}+\mathrm{C}$ and $\mathrm{C}+\mathrm{C}+\mathrm{CAT}$ groups; however, the intracellular ROS levels were increased $(\mathrm{P}<0.05)$ in the control and CAT groups when compared to 0 hour.

Intracellular GSH levels (expressed as arbitrary fluorescence units; Fig. 3) for the control oocytes $(0.24 \pm 0.02)$ were similar $(\mathrm{P}>0.05)$ to those for oocytes treated with the

Table 1

Meiosis stages after in vitro maturation of bovine oocytes in the presence of antioxidants.

\begin{tabular}{llrrl}
\hline Group & Total oocytes & \multicolumn{1}{l}{ GV (\%) } & \multicolumn{1}{c}{ INT (\%) } & \multicolumn{1}{l}{ MII (\%) } \\
\hline Control & 120 & $10.9 \pm 2.0$ & $12.3 \pm 3.3$ & $76.7 \pm 1.7$ \\
C + C & 110 & $14.1 \pm 5.6$ & $5.5 \pm 1.8$ & $80.3 \pm 4.1$ \\
CAT & 104 & $9.4 \pm 5.6$ & $9.9 \pm 4.1$ & $80.6 \pm 5.2$ \\
C + C + CAT & 102 & $10.6 \pm 5.4$ & $11.1 \pm 4.7$ & $78.2 \pm 1.1$ \\
\hline
\end{tabular}

Oocytes were matured for $22 \mathrm{~h}$ in medium supplemented with $0.6 \mathrm{mM}$ cysteine and $100 \mu \mathrm{M}$ cysteamine $(\mathrm{C}+\mathrm{C}) ; 100$ UI catalase (CAT); $0.6 \mathrm{mM}$ cysteine, $100 \mu \mathrm{M}$ cysteamine, and $100 \mathrm{UI}$ catalase $(\mathrm{C}+\mathrm{C}+\mathrm{CAT})$; or received no supplementation (Control). Data are expressed as the mean \pm standard error of five independent replicates.

No differences were observed between treatments ( $P>0.05$, Tukey's test). Abbreviations: GV, germinal vesicle; INT, intermediate stages of meiosis between GV and MII (metaphase I, anaphase I, and telophase I); MII, metaphase II. antioxidants CAT $(0.28 \pm 0.03)$ and $\mathrm{C}+\mathrm{C}+\mathrm{CAT}$ $(0.17 \pm 0.01)$, but $C+C$ group $(0.51 \pm 0.05)$ exhibited greatest $(\mathrm{P}<0.05) \mathrm{GSH}$ levels among all the groups. We found a significant reduction $(\mathrm{P}<0.05)$ in intracellular $\mathrm{GSH}$ levels of the mature oocytes from all the experimental groups compared with the immature oocytes ( 0 hour; $1.00 \pm 0.08)$.

The mitochondrial membrane potential (expressed as arbitrary fluorescence units; Fig. 4) for control oocytes $(1.60 \pm 0.05)$ was greater $(\mathrm{P}<0.05)$ than for the oocytes treated with the antioxidants $C+C(0.94 \pm 0.03)$ and CAT ( $1.41 \pm 0.0)$, yet it was lower $(\mathrm{P}<0.05)$ than for the oocytes treated with $\mathrm{C}+\mathrm{C}+\mathrm{CAT}(1.81 \pm 0.07)$. Overall, $\Delta \Psi \mathrm{m}$ was improved $(\mathrm{P}<0.05)$ in mature oocytes from all groups compared to immature oocytes (0-hour group; $1.00 \pm 0.05)$, with the exception of the $C+C$ group, which did not differ from the 0 -hour group $(\mathrm{P}>0.05)$.

The effects of supplementation with antioxidants during IVM on cleavage and blastocyst rates are given in Figure 5. There was no effect $(P>0.05)$ of antioxidant supplementation on cleavage rates (76.5\%; averaged) or in blastocyst rates (36.1\%; averaged), but there was a tendency $(\mathrm{P}=0.0689)$ to obtain a higher blastocyst rate for the $\mathrm{C}+\mathrm{C}$ + CAT group $(47.5 \pm 5.6 \%)$ compared to control group $(29.9 \pm 4.8 \%)$.

\section{Discussion}

To evaluate the effects of intracellular and/or extracellular antioxidants on the oocyte quality and competence, specific parameters of cytoplasmic maturation (namely GSH and ROS levels as well as $\Delta \Psi \mathrm{m}$ ) were assessed in oocytes that were cultured under standard IVM conditions. The principal results reported here suggest the following: (1) supplementation of IVM medium with intracellular (cysteine and cysteamine) and/or extracellular (catalase) antioxidants reduced intracellular ROS levels in oocytes; (2) supplementation of IVM medium with intracellular antioxidants (cysteine and cysteamine) increased intracellular GSH levels in oocytes; (3) $\Delta \Psi$ m was reduced in oocytes from C + C and CAT treatments; (4) supplementation of IVM medium with a combination of intracellular and extracellular antioxidants $(C+C+C A T)$ increased $\Delta \Psi \mathrm{m}$ in oocytes and tended to increase the blastocyst formation rate.

At the end of IVM, intracellular ROS levels in the control group were increased compared to the levels observed in immature oocytes. During IVM, the formation of certain levels of ROS can increase the developmental potential of oocytes to produce embryos, suggesting that ROS may play different roles depending on both the time they are present throughout the IVC of bovine oocytes and the quantity present [26]. However, a precise balance between ROS production and the cellular antioxidant defense mechanisms is essential for favorable IVC conditions. If this balance is not met, oxidative stress could result in cellular damage, particularly by disrupting the mitochondrial membranes, which ultimately culminates in apoptosis and failed embryonic development [16]. Nevertheless, intracellular ROS levels at the end of maturation were lower in all the treated groups (cysteine and cysteamine association, 

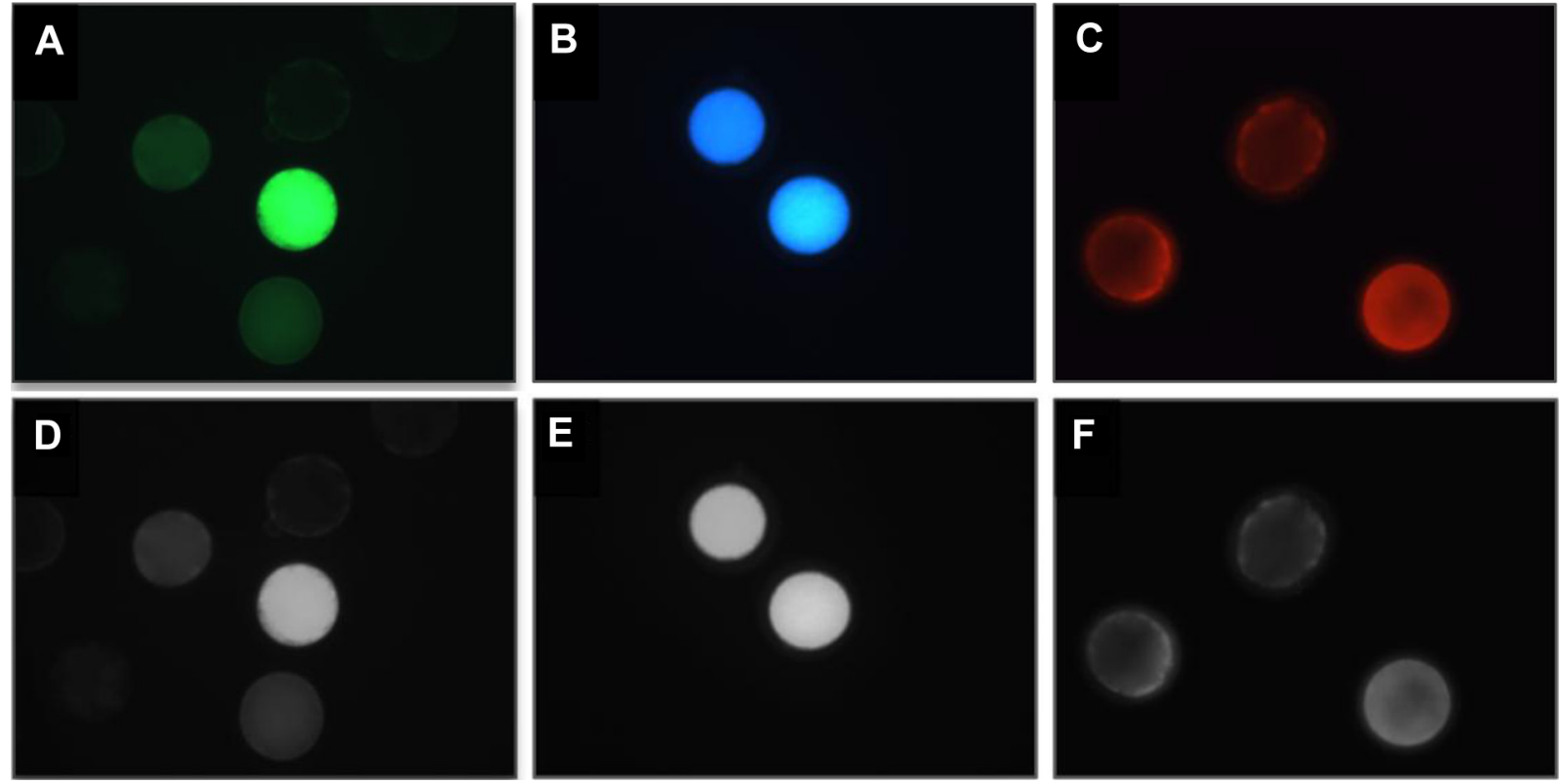

Fig. 1. Representative photomicrographs of bovine oocytes stained for reactive oxygen species, glutathione, and mitochondrial membrane potential $(\Delta \Psi \mathrm{m})$ under fluorescence (A, B, and C) and after conversion to a gray-scale image (D, E, and F). $\times 40$ Magnification.

catalase alone, or even the combination of these three antioxidants) than those in the control group. These effects were expected and are likely due to the action of catalase in removing $\mathrm{H}_{2} \mathrm{O}_{2}$ produced in the extracellular environment (i.e., the culture medium). Intracellular antioxidants (cysteine combined with cysteamine) scavenge the intracellular ROS produced in oocytes and cumulus cells because of the use of oxygen for energy production through mitochondrial oxidative phosphorylation [5,13]. Consequently, the expected effect was a reduction in the inherently deleterious effects due to the action of $\mathrm{H}_{2} \mathrm{O}_{2}$ and other ROS [8], namely, the oxidative modification of cellular components, such as DNA, lipids, and proteins [13]. Interestingly,

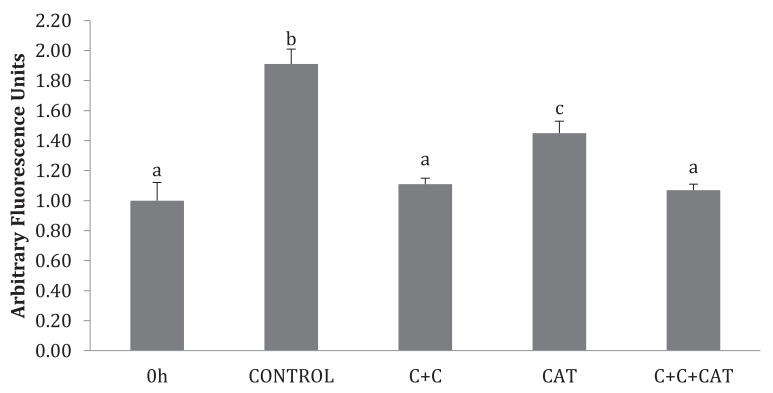

Fig. 2. Effect of antioxidant supplementation during in vitro maturation on intracellular reactive oxygen species levels (expressed as arbitrary fluorescence units) in bovine oocytes. Oocytes were matured for 22 hours in vitro in medium supplemented with $0.6 \mathrm{mM}$ cysteine and $100 \mu \mathrm{M}$ cysteamine $(\mathrm{C}+\mathrm{C} ; \mathrm{n}=110$ ); 100 UI catalase (CAT; $\mathrm{n}=103$ ); $0.6 \mathrm{mM}$ cysteine, $100 \mu \mathrm{M}$ cysteamine, and 100 UI catalase $(C+C+C A T ; n=112)$; or that received no supplementation (control; $\mathrm{n}=111$ ). One group of oocytes was evaluated immediately after their removal from the follicle ( 0 hour; $n=107)$. Treatments were repeated in five replicates. The data represent the mean \pm standard error; different letters indicate significant differences between treatments $(\mathrm{P}<0.05$, Tukey's test). the utilization of catalase seems to be a strategy to allow the production of intermediate levels of intracellular ROS at the end of maturation because accumulating evidence indicates that ROS can regulate cell function both by controlling the production and activation of substances that have biological activity and by activating key downstream signaling pathways [27-29]. Thus, catalase could protect against the harmful effects of high ROS levels yet, at the same time, could use of the reactive nature of ROS for beneficial purposes. However, such putative beneficial effects remain to be investigated.

The synthesis of intracellular GSH is a critical part of oocyte cytoplasmic maturation [30], and the presence of

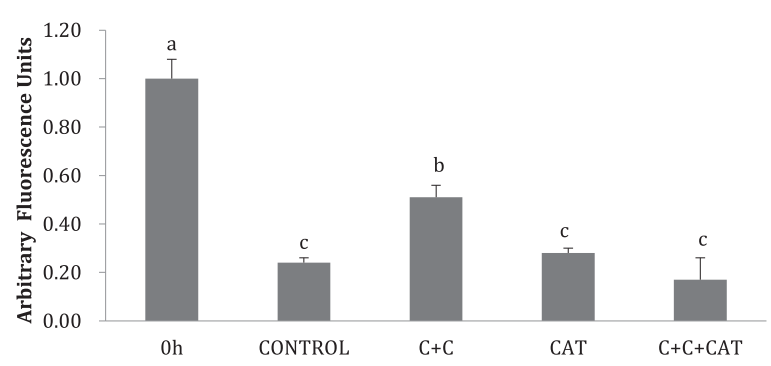

Fig. 3. Effect of antioxidant supplementation during in vitro maturation on intracellular glutathione levels (expressed as arbitrary fluorescence units) in bovine oocytes. Oocytes were matured for 22 hours in vitro in medium supplemented with $0.6 \mathrm{mM}$ cysteine and $100 \mu \mathrm{M}$ cysteamine $(\mathrm{C}+\mathrm{C}$; $\mathrm{n}=111$ ); 100 UI catalase (CAT; $\mathrm{n}=117$ ); $0.6 \mathrm{mM}$ cysteine, $100 \mu \mathrm{M}$ cysteamine, and 100 UI catalase ( $C+C+C A T ; n=130)$; or that received no supplementation (control; $\mathrm{n}=129$ ). One group of oocytes was evaluated immediately after their removal from the follicle ( 0 hour; $n=132$ ). Treatments were repeated in five replicates. The data represent the mean \pm standard error; different letters indicate differences between treatments ( $\mathrm{P}<0.05$, Tukey's test). 


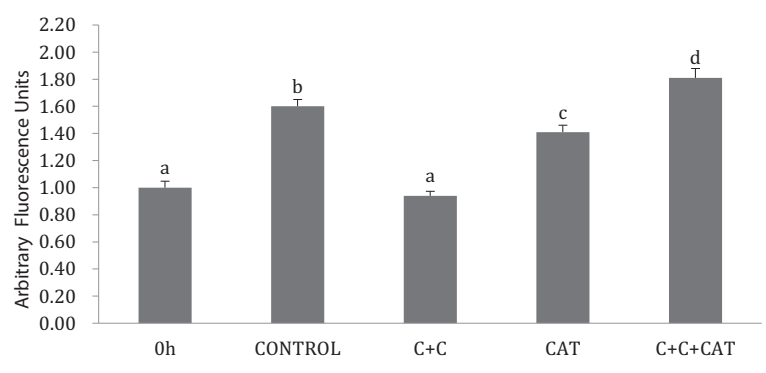

Fig. 4. Effect of antioxidant supplementation during in vitro maturation on mitochondrial membrane potential (expressed as arbitrary fluorescence units) in bovine oocytes. Oocytes were matured for 22 hours in vitro in medium supplemented with $0.6 \mathrm{mM}$ cysteine and $100 \mu \mathrm{M}$ cysteamine $(\mathrm{C}+\mathrm{C} ; \mathrm{n}=130$ ); 100 UI catalase (CAT; $\mathrm{n}=130) ; 0.6 \mathrm{mM}$ cysteine, $100 \mu \mathrm{M}$ cysteamine, and 100 UI catalase $(C+C+C A T ; n=130)$; or that received no supplementation (control; $n=130$ ). One group of oocytes was evaluated immediately after their removal from the follicle ( 0 hour; $n=107$ ). Treatments were repeated in five replicates. The data represent the mean \pm standard error; different letters indicate differences between treatments ( $\mathrm{P}<0.05$, Tukey's test).

high levels of GSH in oocytes at the end of maturation is considered a biochemical marker for improved oocyte quality [31] as this reservoir will protect the zygote and early embryo against oxidative damage before genomic activation and de novo GSH synthesis [13]. However, the results of the present study demonstrated depletion in the amounts of the intracellular GSH in mature oocytes among all the treated groups compared to immature oocytes. An explanation for this finding is that IVC conditions result in higher mobilization and utilization of intracellular GSH in an effort to neutralize the high and continuous amounts of ROS produced by the cells or even in the culture medium $[23,32,33]$. Interestingly, comparison of the in vitromatured oocytes revealed that GSH depletion was lower

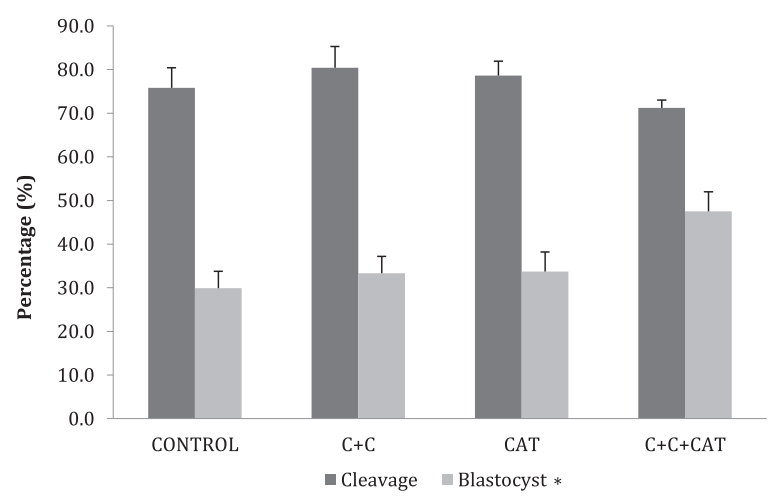

Fig. 5. Effect of antioxidant supplementation during in vitro maturation on cleavage rates and embryo development to the blastocyst stage. Embryos were produced by in vitro fertilization of oocytes that were matured for 22 hours in vitro in medium supplemented with $0.6 \mathrm{mM}$ cysteine and $100 \mu \mathrm{M}$ cysteamine $(\mathrm{C}+\mathrm{C} ; \mathrm{n}=197) ; 100$ UI catalase (CAT; $\mathrm{n}=183$ ); $0.6 \mathrm{mM}$ cysteine, $100 \mu \mathrm{M}$ cysteamine, and 100 UI catalase ( $+C+C A T ; n=195)$; or that received no supplementation (control; $\mathrm{n}=191$ ). Treatments were repeated in five replicates. The data represent the mean \pm standard error. No differences were observed between treatments ( $P>0.05$, Tukey's test). *A tendency to obtain a higher blastocyst rate for $\mathrm{C}+\mathrm{C}+$ CAT group compared to the control group ( $\mathrm{P}=0.0689$ ) was observed. when the oocytes matured in the presence of the thiol compounds, cysteine and cysteamine $(C+C$ group), which are precursors for the synthesis of reduced GSH. Thus, because intracellular GSH synthesis was stimulated by these thiol compounds, the depletion of GSH at the end of IVM was lower in such oocytes. Conversely, catalase did not attenuate the decrease in GSH levels in the in vitromatured oocytes because this extracellular antioxidant enzyme does not stimulate de novo GSH synthesis. Catalase cannot cross the plasma membrane, so this antioxidant does not act through the removal of the $\mathrm{H}_{2} \mathrm{O}_{2}$ produced by the cell $[5,34]$. Instead, it acts by modulating the ROS produced by the culture medium [5,9]. Unexpectedly, intracellular GSH depletion in the oocytes that were matured in the presence of intracellular antioxidants was increased when catalase was associated with the treatment $(\mathrm{C}+\mathrm{C}$ vs. $\mathrm{C}+\mathrm{C}+\mathrm{CAT}$ groups). A reasonable explanation for this finding is that highest $\Delta \Psi \mathrm{m}$ was found in the oocytes from the $\mathrm{C}+\mathrm{C}+\mathrm{CAT}$ group at the end of maturation. As is widely known, increased mitochondrial activity leads to an increase in the exchange of electrons in the inner mitochondrial membrane, which is considered one of the main sources of ROS production [35]. Despite such a high $\Delta \Psi \mathrm{m}$ (discussed in later sections), the intracellular ROS levels in the mature oocytes from the $\mathrm{C}+\mathrm{C}+\mathrm{CAT}$ group were lower than those observed in the oocytes from the control group, suggesting that GSH was consumed to avoid the harmful effects of the high levels of ROS. Therefore, the results presented here indicate that the oxidative stress induced by both the intracellular metabolic reactions and the extracellular medium needs to be considered to fully understand the redox metabolism in cells under culture conditions.

The concept of developmental competence remains illdefined [36], but the mitochondria may play an important role in this competence [37] because oocyte quality is associated with high mitochondrial metabolic status, which is commonly assessed by the $\Delta \Psi \mathrm{m}$ and cellular ATP content. With the exception of the $C+C$ group, the results obtained in this study demonstrated that mature oocytes exhibited higher $\Delta \Psi \mathrm{m}$ compared to immature oocytes. This result is supported by the literature as previous studies have revealed that few immature oocytes display mitochondrial activity, which is probably due to the immaturity of the mitochondria. In contrast, after the IVM of oocytes, mitochondrial activity increases, and the mitochondria become diffusely distributed throughout the cytoplasm [38-40]. According to Tarazona et al. [41], high levels of mitochondrial activity are necessary for further maturation events that are dependent on ATP generation, such as maturation of the nucleus and the completion of meiosis II $[42,43]$, maturation of the cytoplasm [43], rearrangement of the cytoskeleton $[44,45]$, and for the accumulation of the mRNAs that are necessary for early development before the onset of embryonic transcription $[1,43,46]$. Moreover, the mitochondria that exist within the oocyte must provide adequate ATP to fuel the first few days of embryonic development $[47,48]$ because mtDNA replication and the proliferation of mitochondria do not occur until the blastocyst stage [49]. However, it is also assumed that both anaerobic and aerobic respirations attempt to meet the energy needs of the early embryo [50]. Because we did not 
measure ATP content, we cannot correlate the low $\Delta \Psi \mathrm{m}$ in oocytes from the $C+C$ group with a low energy supply, but we assume that ATP levels were not below the minimum level because some of these ATP-dependent events were properly completed in this study (e.g., the completion of meiosis II and embryonic development to the blastocyst stage).

In the present study, improvements in oocyte quality were not reflected by increased embryo production. Findings regarding the inclusion of antioxidants are controversial: several reports demonstrate improvements after the inclusion of antioxidants during IVM [21,51], whereas other studies have reported that antioxidant supplementation during IVM does not influence the rates of cleavage and embryo development to the blastocyst stage $[5,20]$, although some have reported increased intracellular GSH levels [21], decreased ROS levels, and decreased percentage in apoptotic cells [5]. Therefore, results from the literature are inconsistent, and it appears that beneficial effects are only observed when there are inadequate culture conditions [52], which was not the case in the present study because the percentages of obtained embryos were appropriate (36.1\%, averaged). Moreover, SOF medium [53] contains a variety of compounds with antioxidant capacity, such as methionine, hypotaurine, cystine, tyrosine, tryptophan, fetal bovine serum, BSA, pyruvate, and citrate $[8,54]$. In addition, the embryos were cultured on a monolayer of cumulus cells. All these factors may have masked the potential effects of antioxidants during IVM, as suggested previously by Thompson et al. [55].

Although supplementation with antioxidants during IVM did not affect embryo development, the tendency in obtaining a higher blastocyst rate was observed in $\mathrm{C}+\mathrm{C}+$ CAT in comparison with the control group. As mentioned previously, we found that mature oocytes from this group showed a reduction in the intracellular ROS levels even in a condition of increased mitochondrial membrane potential, which may provide higher levels of ATP to oocytes, allowing them to be more competent in sustaining subsequent embryonic development [38]. However, such oocytes did not exhibit the highest concentrations of GSH at the end of IVM, and as it is widely known, the redox status of GSH and other thiols has long been recognized to be critical for the proper mitochondrial function and activity [22]. Concerning oocytes and embryos, mitochondrial function is an important parameter used to assess competency because of the well-known role of mitochondria as the energy/ATP resource for most of the reactions that occur in cells: their metabolic pathways, including the Krebs cycle, fatty acid metabolism, urea metabolism, and certain hormonal metabolisms; their function as cell-death/cell-life mediators; and their function as a storage receptacle for ions and other cofactors that are important for cell homeostasis and regulation $[41,56]$. Thus, the present results suggest that the use of only a single measure to evaluate oocyte quality is not an appropriate way to predict the potential for embryonic development.

In conclusion, our results demonstrate that supplementation of IVM medium with antioxidants improved the quality of oocytes in different ways: (1) decreasing ROS content in oocytes (as demonstrated in supplementation with all types of antioxidants, irrespective of its intracellular or extracellular action); (2) increasing GSH content in oocytes (intracellular antioxidants); and (3) increasing the mitochondrial membrane potential (intracellular combined with extracellular antioxidants). However, despite improvements in the quality of oocytes, the embryonic development to the blastocyst stage was unaffected.

\section{Acknowledgments}

The present study was supported by São Paulo Research Foundation (FAPESP), Brazil (\#2013/07382-6). N.A.S.R.-F. was supported by a PhD scholarship from FAPESP, Brazil (\#2012/10883-8) and G.Z.M. was awarded with scholarship from Conselho Nacional de Desenvolvimento Científico e Tecnológico (CNPq), Brazil (\#306746/2012-3). The authors thank Adão A. Custodio and Alexandre J. Teixeira for technical support.

\section{References}

[1] Trounson A, Anderiesz C, Jones G. Maturation of human oocytes in vitro and their developmental competence. Reproduction 2001; 121:51-75.

[2] Ferreira EM, Vireque AA, Adona PR, Meirelles FV, Ferriani RA, Navarro PAAS. Cytoplasmic maturation of bovine oocytes: structural and biochemical modifications and acquisition of developmental competence. Theriogenology 2009;71:836-48.

[3] Mingoti GZ, Caiado Castro VS, Méo SC, Barretto LS, Garcia JM. The effect of interaction between macromolecule supplement and oxygen tension on bovine oocytes and embryos cultured in vitro. Zygote 2009;17:321-8.

[4] Mingoti GZ, Castro VS, Méo SC, Sá Barretto LS, Garcia JM. The effects of macromolecular and serum supplements and oxygen tension during bovine in vitro procedures on kinetics of oocyte maturation and embryo development. In Vitro Cell Dev Biol Anim 2011;47:361-7.

[5] Rocha-Frigoni NAS, Leão BC, Nogueira É, Accorsi MF, Mingoti GZ. Reduced levels of intracellular reactive oxygen species and apoptotic status are not correlated with increases in cryotolerance of bovine embryos produced in vitro in the presence of antioxidants. Reprod Fertil Dev 2014;26:797-805.

[6] Rocha-Frigoni NAS, Leão BCS, Nogueira É, Mingoti GZ. Effect of gaseous atmosphere and antioxidants on the development and cryotolerance of bovine embryos at different periods of in vitro culture. Zygote 2015;23:159-68.

[7] Corrêa GA, Rumpf R, Mundim TCD, Franco MM, Dode MAN. Oxygen tension during in vitro culture of bovine embryos: effect in production and expression of genes related to oxidative stress. Anim Reprod Sci 2008;104:132-42.

[8] Guérin P, Mouatassim SEL, Ménézo Y. Oxidative stress and protection against reactive oxygen species in the pre-implantation embryo and its surroundings. Hum Reprod Update 2001;7:175-89.

[9] Martín-Romero FJ, Miguel-Lasobras EM, Domínguez-Arroyo JA, González-Carrera E, Álvarez IS. Contribution of culture media to oxidative stress and its effects on human oocytes. Reprod Biomed Online 2008;17:652-61.

[10] Feugang JM, De Roover R, Moens A, Léonard S, Dessy F, Donnay I. Addition of $\beta$-Mercaptoethanol or Trolox at the morula/blastocyst stage improves the quality of bovine blastocysts and prevents induction of apoptosis and degeneration by prooxidants agents. Theriogenology 2004;63:71-90.

[11] Berhman HR, Kodaman PH, Preston SL, Gao S. Oxidative stress on the ovary. J Soc Gynecol Investig 2001;8:40-2.

[12] Gonçalves FS, Barreto LSS, Arruda RP, Perri SHV, Mingoti GZ. Effect of antioxidants during bovine in vitro fertilization procedures on spermatozoa and embryo development. Reprod Domest Anim 2010; 45:129-35.

[13] Deleuze S, Goudet G. Cysteamine supplementation of in vitro maturation media: a review. Reprod Domest Anim 2010;45:476-82.

[14] Bain NT, Madan P, Betts DH. The early embryo response to intracellular reactive oxygen species is developmentally regulated. Reprod Fertil Dev 2011;23:561-75. 
[15] Pierce JD, Cackler AB, Arnett MG. Why should you care about free radicals? RN 2004;67:38-42.

[16] Tvrdá E, Knazická Z, Bárdos 1, Massányi P, lukác N. Impact of oxidative stress on male fertility - a review. Acta Vet Hung 2011;59: 465-84.

[17] Kitagawa Y, Suzukib K, Yonedaa A, Watanabea T. Effects of oxygen concentration and antioxidants on the in vitro developmental ability, production of reactive oxygen species (ROS), and DNA fragmentation on porcine embryos. Theriogenology 2004;62:1186-283.

[18] Johnson MH, Nars-Esfahani MH. Radical solutions and cultural problems: could free oxygen radicals be responsible for the impaired development of preimplantation mammalian embryos in vitro? Bioessays 1994;16:31-9.

[19] Orsi NM, Leese HJ. Protection against reactive oxygen species during mouse preimplantation embryo development: role of EDTA, oxygen tension, catalase, superoxide dismutase and pyruvate. Mol Reprod Dev 2001;59:44-53.

[20] Ali AA, Bilodeau JF, Sirard MA. Antioxidant requirements for bovine oocytes varies during in vitro maturation, fertilization and development. Theriogenology 2003;59:939-49.

[21] De Matos DG, Furnus CC. The importance of having high glutathione (GSH) levels after bovine in vitro maturation on embryo development: effect of $\beta$-mercaptoethanol, cysteine and cystine. Theriogenology 2000;53:761-842.

[22] Lash LH. Mitochondrial glutathione transport: physiological, pathological and toxicological implication. Chem Biol Interact 2006;163: 54-67.

[23] Curnow EC, Ryan J, Saunders D, Hayes ES. Bovine in vitro oocyte maturation as a model for manipulation of the $\gamma$-glutamyl cycle and intraoocyte glutathione. Reprod Fertil Dev 2008;20:579-88.

[24] Parrish JJ, Susko-Parrish J, Winer MA, First NL. Capacitation of bovine sperm by heparin. Biol Reprod 1988;38:1171-88.

[25] Vajta G, Rindom N, Peura TT, Holm P, Greve T, Callesen H. The effect of media, serum and temperature on in vitro survival of bovine blastocysts after open pulled straw (OPS) vitrification. Theriogenology 1999;52:939-48.

[26] Blondin P, Coenen K, Sirard MA. The impact of reactive oxygen species on bovine sperm fertilizing ability and oocyte maturation. J Androl 1997; 18:454-60.

[27] Buhimschi IA, Kramer WB, Buhimschi CS, Thompson LP, Weiner CP. Reduction-oxidation (redox) state regulation of matrix metalloproteinase activity in human fetal membranes. Am J Obstet Gynecol 2000;182:458-64.

[28] Marshall HE, Merchant K, Stamler JS. Nitrosation and oxidation in the regulation of gene expression. FASEB J 2000;14:1889-900.

[29] Morado SA, Cetica PD, Beconi MT, Dalvit GC. Reactive oxygen species in bovine oocyte maturation in vitro. Reprod Fertil Dev 2009;21: $608-14$.

[30] Eppig JJ. Coordination of nuclear and cytoplasmic oocyte maturation in eutherian mammals. Reprod Fertil Dev 1996;8:485-9.

[31] De Matos DG, Furnus CC, Moses DF, Baldassare H. Effect of cysteamine on glutathione level and developmental capacity of bovine oocytes matured in vitro. Mol Reprod Dev 1995;42:432-8.

[32] Livingston T, Rich K, Mackensie S, Godkin JD. Glutathione content and antioxidant enzyme expression of in vivo matured sheep oocytes. Anim Reprod Sci 2009;116:265-73.

[33] Cetica PD, Pintos LN, Dalvit GC, Beconi MT. Antioxidant enzyme activity and oxidative stress in bovine oocyte in vitro maturation. IUBMB Life 2001;51:57-64.

[34] Kouridakis K, Gardner DK. Pyruvate in embryo culture media acts as an antioxidant. Proc Fertile Soc Aus 1995;4:29.

[35] Zhao X, Du W, Wang D, Hao H, Liu Y, Qin T, et al. Recovery of mitochondrial function and endogenous antioxidant systems in vitrified bovine oocytes during extended in vitro culture. Mol Reprod Dev 2011;78:942-50.
[36] Duranthon V, Renard J. The developmental competence of mammalian oocytes: a convenient but biologically fuzzy concept. Theriogenology 2001;55:1277-89.

[37] Cummins JM. Cytoplasmic inheritance and its implications for animal biotechnology. Theriogenology 2001;55:1381-99.

[38] Stojkovic M, Machado SA, Stojkovic P, Zakhartchenko V, Hutzler P, Gonçalves PB, et al. Mitochondrial distribution and adenosine triphosphate content of bovine oocytes before and after in vitro maturation: correlation with morphological criteria and developmental capacity after in vitro fertilization and culture. Biol Reprod 2001;64:904-9.

[39] Wilding M, Dale B, Marino M, di Matteo L, Alviggi GP, Isaruto ML, et al. Mitochondrial aggregation patterns and activity in human oocytes and preimplantation embryos. Hum Reprod 2001;16:909-17.

[40] Tamassia M, Nuttinck F, May-Panloup P. In vitro embryo production efficiency in cattle and its association with oocyte adenosine triphosphate content, quantity of mitochondrial DNA, and mitochondrial DNA haplogroup. Biol Reprod 2004;71:697-704.

[41] Tarazona AM, Rodriguez JI, Restrepo LF, Olivera-Angel M. Mitochondrial activity, distribution and segregation in bovine oocytes and in embryos produced in vitro. Reprod Domest Anim 2006;41: $5-11$.

[42] de Vantery C, Stutz A, Vassali JD, Schoderet-Slatkine S. Acquisition of meiotic competence in growing mouse is controlled at both translational and posttranslational levels. Dev Biol 1997;187:43-54.

[43] Picton H, Briggs D, Gosden R. The molecular basis of oocyte growth and development. Mol Cell Endocrinol 1998;145:27-37.

[44] Wang T, Lessman CA. Isoforms of soluble alpha-tubulin in oocytes and brain of the frog (genus Rana): changes during oocyte maturation. Cell Mol Life Sci 2002;59:2216-23.

[45] Van Blerkom J, Davis P, Alexander S. Differential mitochondrial distribution in human pronuclear embryos leads to disproportionate inheritance between blastomeres: relationship to microtubular organization, ATP content and competence. Hum Reprod 2000;15:2621-33.

[46] Gary DS. In vitro maturation of oocytes. Curr Womens Health Rep $2001 ; 1: 143-51$.

[47] Thouas GA, Trounson AO, Jones GM. Effect of female age on mouse oocyte developmental competence following mitochondrial injury. Biol Reprod 2005;73:366-73.

[48] Lin DPC, Huang CC, Wu HM, Cheng TC, Chen CI, Lee MS. Comparison of mitochondrial DNA contents in human embryos with good or poor morphology at the 8-cell stage. Fertil Steril 2004;81:73-9.

[49] Thundathil J, Filion F, Smith Lawrence C. Molecular control of mitochondrial function in preimplantation mouse embryos. Mol Reprod Dev 2005;71:405-13.

[50] Chappel S. The role of mitochondria from mature oocyte to viable blastocyst. Obstet Gynecol Int 2013;2013:1-10.

[51] De Matos DG, Furnus CC, Moses DF, Baldassare H. Cysteamine supplementation during in vitro maturation and embryo culture: a useful tool for increasing the efficiency of bovine in vitro embryo production. Mol Reprod Dev 2002;62:203-9.

[52] Harvey AJ. The role of oxygen in ruminant preimplantation embryo development and metabolism. Anim Reprod Sci 2007;98:113-28.

[53] Vajta G, Rindom N, Peura TT, Holm P, Greve T, Callesen H. The effect of media, serum and temperature on in vitro survival of bovine blastocysts after open pulled straw (OPS) vitrification. Theriogenology 1999;57:1453-65.

[54] Leminska A, Wnuk M, Slota E, Bartosz G. Total antioxidant capacity of cell culture media. Clin Exp Pharmacol Physiol 2007;34:781-6.

[55] Thompson JGE, Simpon AC, Pugh PA, Donnely PE, Tervit HR. Effect of oxygen concentration on in vitro development of preimplantation sheep and cattle embryos. J Reprod Fertil 1990;89:573-8.

[56] Scheffler IE. Mitochondria. First edition. New York: J. Wiley \& Sons, Inc.; 1999. 